\title{
Multi-Channel Monolithic Integrated Optic Fiber Bragg Grating Sensor Interrogator
}

\author{
Edgar A. MENDOZA, Yan ESTERKIN, Cornelia KEMPEN, and Zongjian SUN \\ Redondo Optics Inc., 811 North Catalina Avenue, Suite 1100, Redondo Beach, CA 90277, USA \\ *Corresponding author: Edgar MENDOZAＥ-mail: emendoza@redondooptics.com
}

\begin{abstract}
Fiber Bragg grating (FBG) is a mature sensing technology for the measurement of strain, vibration, acoustics, acceleration, pressure, temperature, moisture, and corrosion. It has gained rapid acceptance in civil, aerospace, chemical and petrochemical, medicine, aviation and automotive industries. The most prominent advantages of FBG are: small size and light weight, distributed array of FBG transducers on a single fiber, and immunity to radio frequency interference. However, a major disadvantage of FBG technology is that conventional state-of-the-art FBG interrogation system is typically bulky, heavy, and costly bench top instruments that are typically assembled from off-the-shelf fiber optic and optical components integrated with a signal electronics board into an instrument console. Based on the industrial need for a compact FBG interrogation system, this paper describes recent progress towards the development of miniature fiber Bragg grating sensor interrogator (FBG-Transceiver ${ }^{\mathrm{TM}}$ ) system based on multi-channel monolithic integrated optic sensor microchip technology. The integrated optic microchip technology enables monolithic integration of all functionalities, both passive and active, of conventional bench top FBG sensor interrogator system, packaged in a miniaturized, low power operation, $2 \mathrm{~cm} \times 5 \mathrm{~cm}$ small form factor (SFF) package suitable for long-term structural health monitoring in applications where size, weight, and power are critical for operation.
\end{abstract}

Keywords: Integrated optics, monolithic, fiber sensors, structural health monitoring, small size, light weight, low power

\section{Introduction}

Infrastructure system is integral to the social, political, and economic wellbeing of a nation. Facets of infrastructure systems affect the quality of buildings and structures, the air we breathe and the water we drink, our access to energy (e.g., electricity, oil, and gas), and communications, inter-modal transportation systems, and disposal of waste. Because these systems are so pervasive, complex, and inherent to our lives, and because of the continuous and ever-increasing threats of environment and global terrorism, they need to be secured and made smart.
In recent years, fiber Bragg grating (FBG) technology has been accepted as a new kind of sensing element for structural health monitoring (SHM) for in situ monitoring of advanced structures in aviation, aerospace systems, civil structures, and petrochemical industry $[1,2,3]$.

The cost of the FBG sensor production, the availability of high-quality and environmentally robust FBG demodulation system as well as practical sensor embedding and packaging techniques are the cores for FBG sensors to be widely popularized for real time monitoring of infrastructure systems [4]. Because of the features of

Received: 15 October 2010 / Revised version: 28 November 2010

(C) The Author(s) 2011.This article is published with open access at Springerlink.com 
lightness, micron-size transducers, and immunity to electromagnetic interference, they can be easily casted, embedded, or surface mounted on a structure. The FBG sensors can be produced in glass or plastic optical fiber to meet harsh environmental conditions and structural demands for a wide variety of applications. The sensor fibers are packaged in strong, rugged materials to withstand harsh environments such as being embedded in fiber composite structures [5], large concrete and metallic structural systems [6], transatlantic fiber cable installations [7], and miniature respiratory monitor systems [8].

However, a major drawback for the global implementation of FBG technology in smart infrastructure applications is that commercially available FBG interrogation units are costly, bench size units, several-pounds of weight, which are too heavy and large to be permanently installed to a critical structure such as airplane wing, rotor blade, or turbine engine [9].

Because of the need for compact, low weight, low power FBG interrogation systems, Redondo Optics, Inc. is currently developing a family of miniature $\mathrm{FBG}$ interrogation (FBG-Transceiver ${ }^{\mathrm{TM}}$ ) systems that use Redondo Optics Inc. (ROI) proprietary integrated optic microchip technology as an optical bench to integrate all the functionalities of the key passive and active optoelectronics components of conventional FBG interrogation systems, such as the light guides, splitters and couplers, light sources, photodetectors, FBG sensor signal demodulators, and signal processing electronics packaged in a miniaturized, environmentally qualified, hermetically sealed single fiber FBG-Transceiver ${ }^{\mathrm{TM}}$ system suitable for the in situ installation and long term operation in civil, petrochemical, and aerospace industrial applications where size, weight, and power consumption are critical requirements for the installation of structural health monitoring of critical infrastructure systems.

\section{FBG-Transceiver ${ }^{\mathrm{TM}}$ system}

The FBG-Transceiver ${ }^{\mathrm{TM}}$ is a miniature, multichannel fiber Bragg grating sensor interrogation system that offers a reliable and cost affordable FBG sensor readout system solution for applications where weight, size, and power are critical for operation $[10,11]$. The FBG-Transceiver ${ }^{\mathrm{TM}}$ system is based on the integration of state-of-the-art monolithic planar lightwave circuit technology with automated telecommunications optical components packaging and complementary metal-oxide semiconductor (CMOS) electronics to enable the design and low cost production of a next-generation, high performance, environmentally robust, miniature FBG sensor interrogation device [12].

Redondo Optics has developed engineering prototypes of a single channel and a multi-channel FBG-Transceiver ${ }^{\mathrm{TM}}$ devices that are currently used for demonstration to selective customers and strategic partners, as shown in Fig. 1.
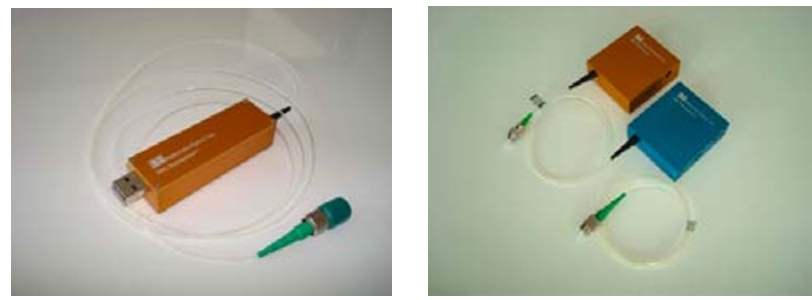

Fig. 1 Single channel and multi-channel FBG-Transceiver ${ }^{\mathrm{TM}}$ devices.

Compared to conventional bench-top FBG interrogation devices, Redondo Optics FBG-Transceiver ${ }^{\mathrm{TM}}$ system has many advantages. These include: 1) there are no moving parts, or complex fiber optic connections that are typically sensitive to motion, shock, vibration, and the moisture and temperature environments; 2) it employs hybrid monolithic integration of all the passive and active optoelectronic components of the system within a miniature optical chip that incorporates passive optical components such as waveguide routers, splitters and couplers, and wavelength spectral demodulation elements hybridly integrated to active components such as light source 
and photodetector arrays, and the hybrid monolithic microchip surface is mounted to a CMOS electronics board that is hermetically sealed in a 2 $\mathrm{cm} \times 5 \mathrm{~cm}$ small form factor (SFF) standard telecommunications package; 3 ) it is produced using standard automated manufacturing practices common in the semiconductor integrated circuit (IC) and telecommunications component industries, which results in a high scale manufacturable low-cost device; 4) the system can be battery operated enabling operation for a period of one year; 5) it uses standard data communication protocols that facilitate integration to any communication network.

Table 1 Features, advantages, and benefits.

\begin{tabular}{l|l|l}
\hline \multicolumn{1}{c|}{ Features } & \multicolumn{1}{|c}{ Advantages } & \multicolumn{1}{|c}{ Benefits } \\
\hline Multichannel & $\begin{array}{l}\text { Multiple sensing points (1 } \\
\text { to 12) distributed along } \\
\text { sensing }\end{array}$ & $\begin{array}{l}\text { Simultaneous health monitoring } \\
\text { thength of a single } \\
\text { atical fiber }\end{array}$ \\
\hline multiple locations of a \\
critical structure
\end{tabular}

Compared to state-of-the-art strain gauge and ultrasound structural health inspection techniques the FBG-TransceiverTM technology offers many advantages as shown in Table 1. These include: 1) all optical sensor transducers are electrically passive; 2) micron size transducers are produced in a 125-micron-diameter optical fiber; 3) it employs simultaneous interrogation of 1 to 12 sensors multiplexed on a single fiber; 4) there is no electromagnetic interference or pick-up; 5) sensors can be surface mounted using conventional strain gauge application techniques, or embedded in composite structures during manufacture; 6) miniature, low weight, and low power consumption sensor read-out unit can be directly installed in structure for the long-term, real-time monitoring; 7) it employs the most affordable structural health sensing technology available in the market today.

The principle of operation of FBG sensor is based on the environmentally induced wavelength shift, associated with changes in stress-strain, temperature, vibration, pressure, etc., of the active peak wavelength of the grating that is attached to the structure under evaluation. ROI uses a passive demodulation technique, based on dispersive filter, in which the wavelength encoded optical signature of the each of the FBG transducers in the array is transformed into an electrical signal at each of the photo receivers by means of the optical properties of the dispersive filter. A microprocessor controller mounted on a CMOS-PC board processes the transformed electrical signal carrying the information from each of the FBG sensors, and transmits the process signals to a remote station via a USB (universal serial bus) data communication interface. The complete electrical power budget for the FBG-Transceiver ${ }^{\mathrm{TM}}$ unit is estimated at approximately less than or equal to $0.1 \mathrm{~W}$, allowing the use of the same USB communication port to provide power to the unit. The complete monolithic integrated optic microchip and signal processing IC-electronic unit is packaged in a single fiber, telecommunications grade, small form factor package to produce a miniature multi-channel FBG-Transceiver ${ }^{\mathrm{TM}}$ system that can be used to monitor the status of FBG transducers embedded or surface mounted on the structure. 


\section{Integrated optic microchip technology}

Planar lightwave circuit (PLC) technology has emerged as a new optical platform of choice for integration and large scale manufacturing of optical components. The technology draws on the excellent heat-dissipation and mechanical properties of silicon wafers. Optical component designers use the silicon substrate as an optical bench to integrate unpackaged optical components, such as lasers, photodiodes, and micro-optic elements in die form, onto PLC chip and then re-package the chip in a single unit. Precise micro-machine features on PLC chip allow passive mechanical alignment and attachment of different opto-electronic components, all accurate enough for reliable communication between the components and the outside world.

The optical communication between the components is accomplished by micron-size, optical waveguide circuits written on a thin film of glass deposited onto a silicon wafer. These waveguide circuits are used to guide light, in a manner similar to optical fiber, through the chip, and perform passive (light guiding) and active (wavelength separation, wavelength filtering, light amplification, switching, modulation, etc.) functions in a manner similar to integrated electronic circuits. Today, high performance PLC chips, as with electronic IC microprocessors, are commercially produced using a variety of mass producible semiconductor technologies. The net result is the integration of sophisticated, multi-element photonic subsystems into mass-produced PLCs.

For the designers of FBG sensor systems, the most exciting feature of integrated optics technology is its influence on the size, weight, and environmental reliability of the device. Freeing all of the optical components from their individual, hermetic package results in 10 times or more reduction in subsystem size and weight. Of course, eliminating excess packaging also reduces the likelihood of subsystem failure because of the interconnect failure.

In its simplest form, ROI's integrated optic microchip integrates a temperature- and power-stabilized broadband semiconductor light source that is monolithically integrated to a PLC microchip to illuminate and interrogate the status of each of the FBG transducers distributed along the sensing fiber. The light source is guided internally through the PLC microchip, using waveguide structures, and coupled to the sensing fiber that connects to the FBG-Transceiver ${ }^{\mathrm{TM}}$ SFF package. Each fiber grating distributed along the sensing fiber reflects a portion of the light source broadband spectrum, determined by Bragg condition of the grating, and transmits the remaining light to the next grating. The returned, wavelength-encoded light signal from each of the distributed FBG sensors is received and processed by the PLC microchip. The received light signal is guided internally through the microchip, using waveguide routing structures, to the photodetectors assigned to monitor a specific wavelength from each of the distributed FBG transducers.

Two types of wavelength division multiplexing (WDM) waveguide architectures are used in the PLC microchip for the spectral separation of the individual wavelengths of each FBG sensors in the fiber sensor array. One type, used with the low channel count $\left(<12\right.$ channels) FBG-Transceiver ${ }^{\mathrm{TM}}$ units, uses wavelength selective dispersive filter structures, and the other type, used with the high channel count ( $>12$ channels) FBG-Transceiver ${ }^{\mathrm{TM}}$ units, uses array waveguide grating (AWG) architectures. The wavelength selective WDM structures allow the transmission of a particular FBG wavelength $\left(\lambda_{1}\right)$ while reflecting, or separating, all of the other $\left(\lambda_{2}, \lambda_{3}, \lambda_{n}\right)$ FBG sensor wavelengths to the respective detection channels.

This process is repeated in a wavelength specific at each photodetector to achieve a wavelength demultiplexing PLC structure. WDM filters are commonly used in long haul fiber optic 
telecommunications networks to either mix (multiplex) or separate (demultiplex) large numbers of communication wavelengths. By carefully selecting the spectral optical bandpass properties of the filter and the peak wavelength shift, environmentally induced, optical signal from each of the FBG sensors is converted into a linear intensity variation, directly related to the physical state (peak wavelength position) of the FBG sensor at the photodetector element, as shown in Fig. 2. This principle forms the basis of the FBG sensor demodulator in the FBG-Transceiver ${ }^{\mathrm{TM}}$ system.

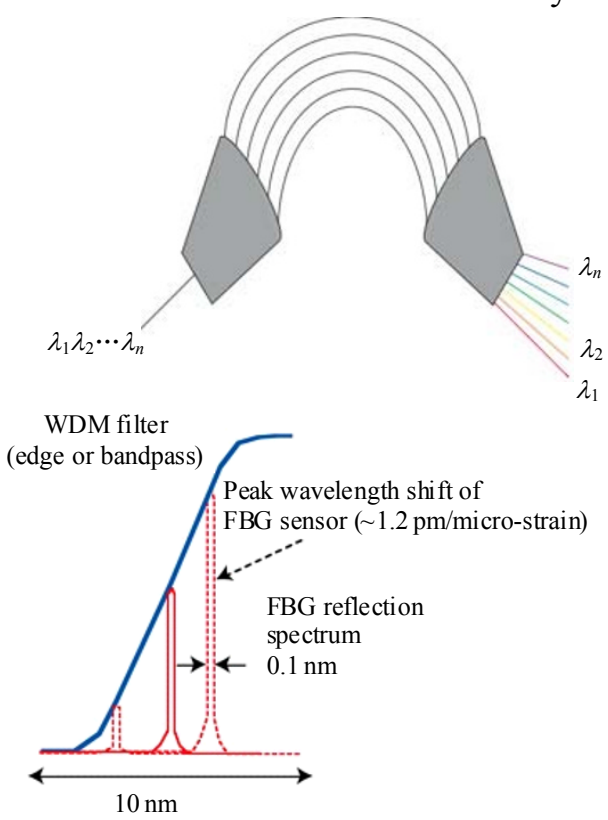

Fig. 2 Integrated broadband and narrowband WDM filter structure for passive wavelength separation and demodulation of FBG sensor signals.

\section{Direct-write integrated optic microchip technology}

Planar lightwave circuits are optical devices fabricated using silicon wafer processing technology. PLCs are typically fabricated on silicon wafers using common semiconductor processes and materials, which make them attractive from a mass manufacturing and cost point of view.

ROI has developed its own well-established PLC fabrication method based on direct-write PSOG ${ }^{\mathrm{TM}}$ technology. ROI's PLC production method is similar

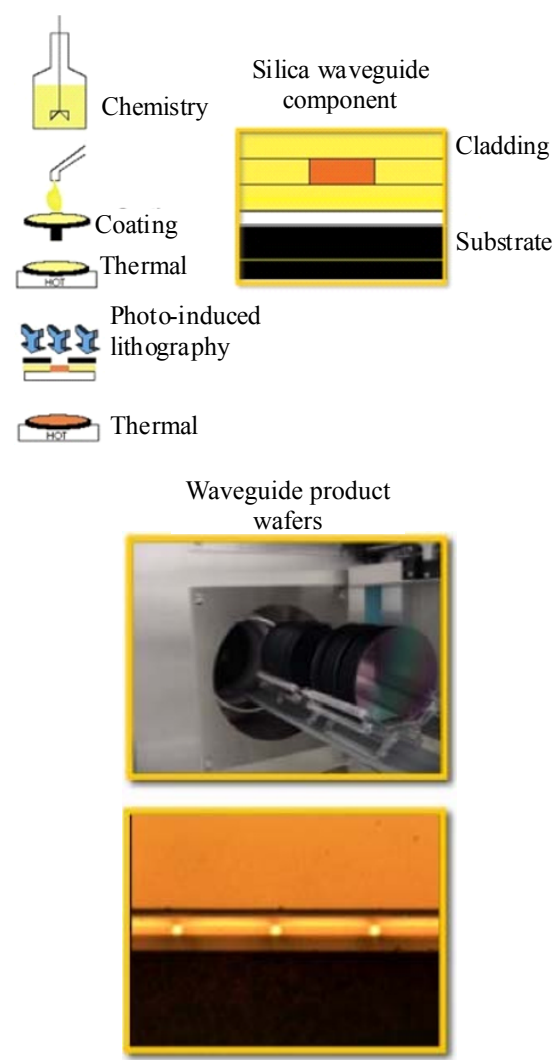

Fig. 3 Planar lightwave circuit fabrication using photosensitive spin-on-glass $\left(\mathrm{PSOG}^{\mathrm{TM}}\right)$ process.

to the SOS technology with two differing steps: 1) deposition of the silica film is achieved via a solution process, and 2) definition of the waveguide structure is achieved by an etchless direct lithography step. ROI's direct-write PSOG $^{\text {TM }}$ process allows the lithographic production of gradient index (GRIN) waveguide structures. GRIN waveguide structures are key in the production of the FBG-Transceiver ${ }^{\mathrm{TM}}$ integrated optic microchip since it allows the fabrication of adiabatic, gradial variations of the refractive index along the propagation plane of the waveguide as well as in the horizontal and vertical planes of the waveguide structures. The GRIN waveguide structures are used to produce the taper mode adapters to enhance the mode coupling efficiency from the light source, a superluminescence light emitting diode (SLED), to the waveguide, and also to produce taper structures used for the WDM wavelength separation and demodulation of the FBG sensor signals. 


\section{Demonstration of operating principle of FBG-Transceiver ${ }^{\mathrm{TM}}$ technology}

To demonstrate the operating principle of the miniature fiber Bragg grating sensor interrogator FBG-Transceiver ${ }^{\mathrm{TM}}$ technology, an engineering prototype unit was developed based on ROI's proprietary multi-channel integrated optic microchip technology.

ROI designed and produced a state-of-the-art signal processing electronic CMOS-PC board used to interrogate the status of the distributed FBG transducers, to demodulate the FBG optical signatures, and to transmit this information via USB data communication to a remote control station. A user-friendly Visual Basic data acquisition software program was developed to display the status of the FBG sensors in real time. The FBG-Transceiver ${ }^{\mathrm{TM}}$ optic block and electronic board were packaged in telecommunications' standard common form (CM) factor $(1.8 \mathrm{~cm} \times 5 \mathrm{~cm} \times 5 \mathrm{~cm})$ single fiber bi-directional transmission package, as shown in Fig. 4.

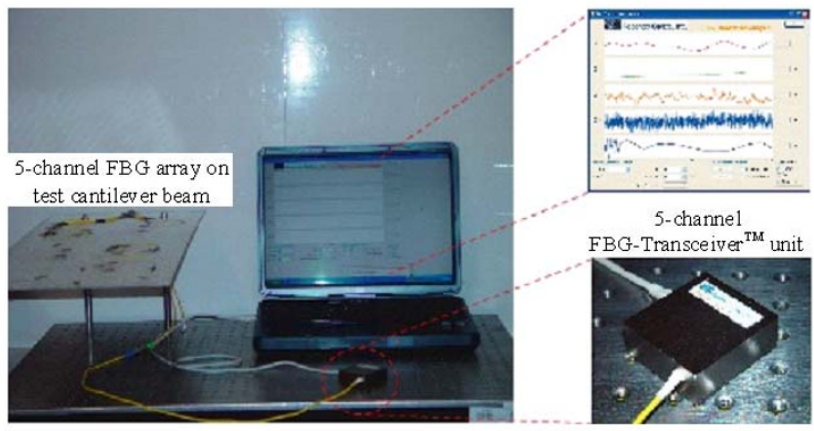

Fig. 4 Miniature multi-channel FBG-Transceiver ${ }^{\mathrm{TM}}$ system.

To test and characterize the performance of the multi-channel FBG-Transceiver ${ }^{\mathrm{TM}}$ prototype, a test bed was assembled by surface mounting an array of five FBG sensors on a cantilever test plate. The cantilever test plate allows inducing variations in the strain state of the FBG sensors as a function of an external induced perturbation. In this case a small progressive, upward or lower, displacement of the cantilever test plate allows monitoring the strain condition of the FBG sensors attached to the structure. To monitor the status of the FBG sensors, the sensor fiber is connected to the multi-channel FBG-Transceiver ${ }^{\mathrm{TM}}$ system using a mating sleeve, and in turn this system is connected to a computer via a USB communication port. A LabView graphical user interface (GUI) is then used to initialize the system and establish communication with the FBG-Transceiver ${ }^{\mathrm{TM}}$ unit. Pressing the run button on the software screen initiates data acquisition and display of the five FBG sensor channels in real time as shown in Fig. 5. Pressing the save button, stores all collected data in a text file.

Figure 5 shows the results of a typical preliminary test in which the experimental test run was initiated driving the cantilever plate steady (flat position), and then a few seconds later the cantilever test plate was bended downwards and hold in a fix bend position for some time. The plate was then released to return to its original flat state. As observed in Fig. 5, all five-sensor channels respond to the downward perturbation of the cantilever test plate in a similar fashion and in real time. It is interesting to observe that one of the FBG sensors experiences a compressive strain (channel 5) as opposed to the other four sensors that experience a tensile strain. This change in the strain behavior of the FBG sensor is related to the position where the sensor was mounted on the cantilever plate.

Using this type of cantilever test bed can calibrate the strain curve of the FBG sensor as a function of an applied load. Typically during calibration experiments, the peak wavelength response of the FBG-Transceiver ${ }^{\mathrm{TM}}$ is correlated with an optical spectrum analyzer for comparison of the peak wavelength detection accuracy. This test setup is used to interrogate the strain status of the FBG sensor array upon bending of the cantilever beam, as shown in Fig. 6. The plot in this figure shows a linear correlation between the peak position of the FBG sensor and the light intensity measured by the demodulation detector channel. The results in 
this plot show a displacement on the peak wavelength position of the grating of about $1.87 \mathrm{~nm}$, corresponding to approximately 1558 microstrains. Measurements of the strain resolution of the FBG-Transceiver ${ }^{\mathrm{TM}}$ system have demonstrated the capability to measure 10-microstrain changes over the range of \pm 2000 microstrains.

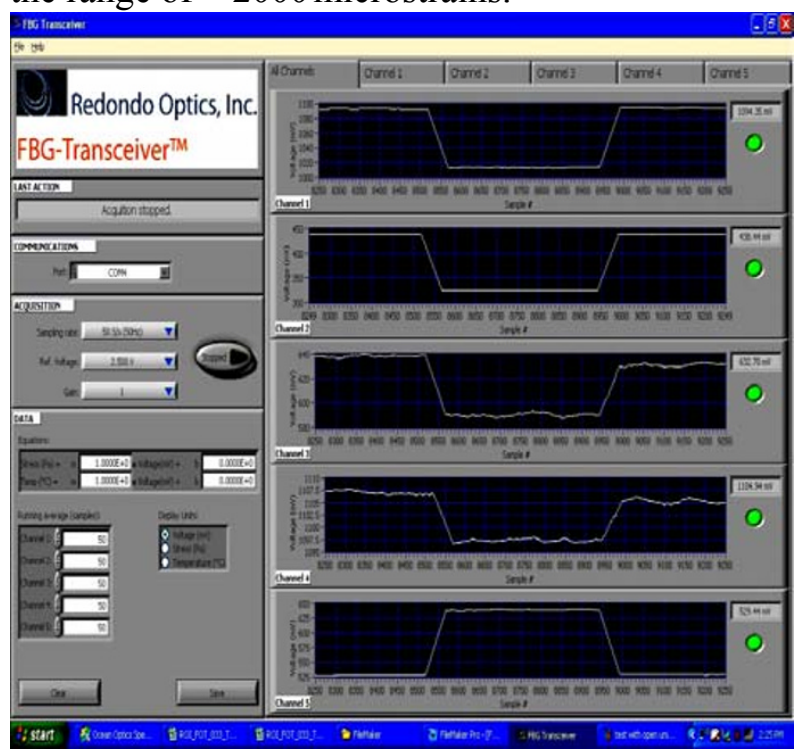

Fig. 5 LabView graphical user interface of FBG-Transceiver ${ }^{\mathrm{TM}}$ system.

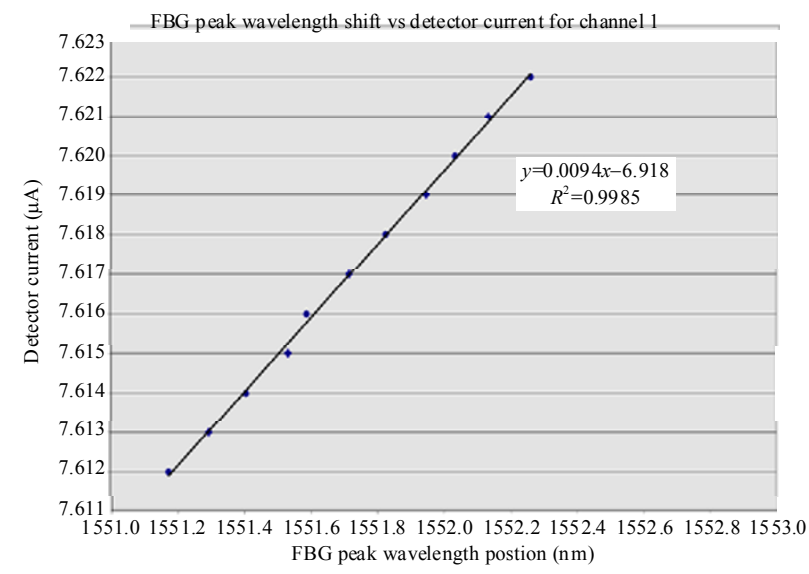

Fig. 6 Passive wavelength demodulation properties of multi-channel FBG-Transceiver ${ }^{\mathrm{TM}}$ system.

Similarly, the dynamic peak wavelength, strain response of the FBG-Transceiver ${ }^{\mathrm{TM}}$ system can be demonstrated by exciting vibration modes on the cantilever beam incorporating the surface-mounted FBG transducers, as shown in Fig. 7.

The vibration of the cantilever plate is clearly monitored in real time using the WDM demodulation principle of the FBG-Transceiver ${ }^{\mathrm{TM}}$ device. The results in Fig. 8 clearly demonstrate the WDM wavelength demodulation principle of the FBG-Transceiver ${ }^{\mathrm{TM}}$ device as it is applied to the interrogation of the wavelength-encoded signal of FBG transducers and the subsequent conversion of their optical signal to an electrical signal for monitoring of the passive and active environmental status of the FBG sensor array in real time.

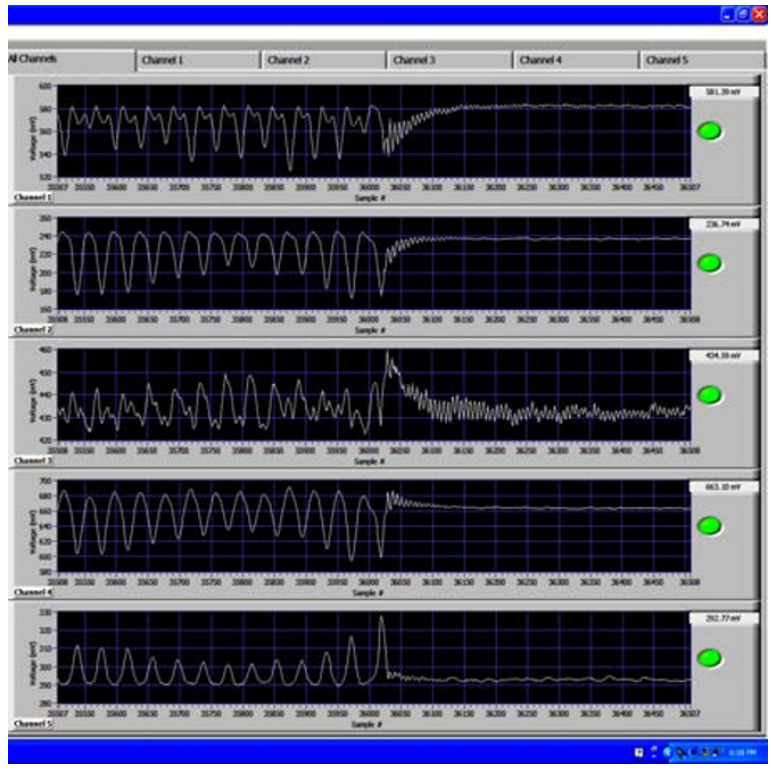

Fig. 7 Dynamic wavelength demodulation properties of multi-channel FBG-Transceiver ${ }^{\mathrm{TM}}$ system.

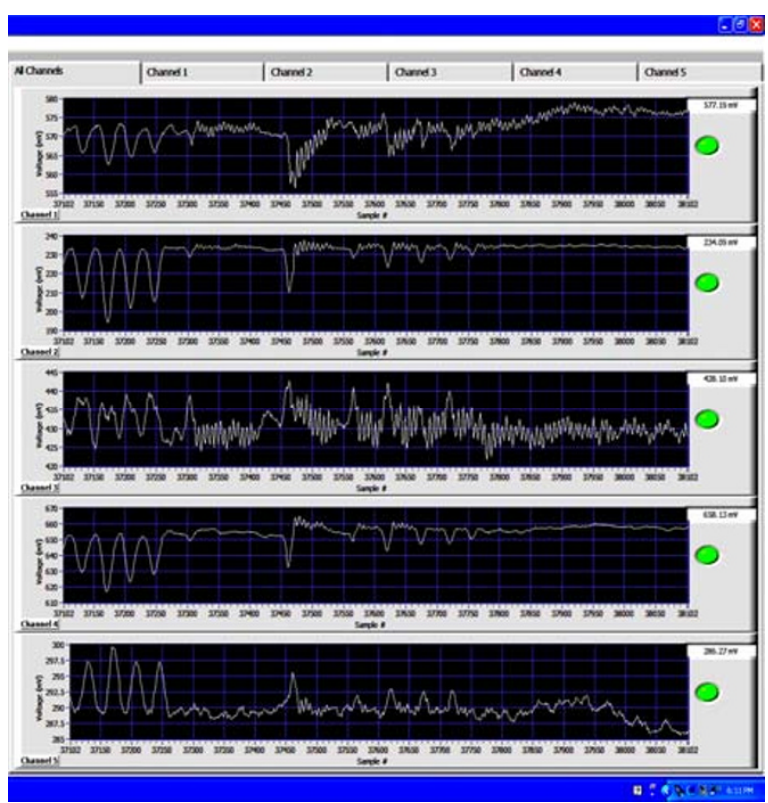

Fig. 8 Passive and dynamic wavelength demodulation properties of multi-channel FBG-Transceiver ${ }^{\mathrm{TM}}$ system. 
Figure 8 further demonstrates a test in which the cantilever plate is randomly excited to passive strain and low and high frequnency vibrations. Currently the FBG-Transceiver ${ }^{\mathrm{TM}}$ operates at frequencies up to $2 \mathrm{kHz}$ simultaneously for all sensor channels. New software is currently in development that will enhance the sampling rate to $20 \mathrm{kHz}$ to enable acoustics detection.

\section{Conclusions}

The results presented in this paper describe on-going work towards the development of a miniature multi-channel fiber Bragg grating sensor interrogator (FBG-Transceiver ${ }^{\mathrm{TM}}$ ) system based on monolithic integrated optic sensor microchip technology. The test results of the FBG-Transceiver ${ }^{\mathrm{TM}}$ device have demonstrated the demodulation principle of the monolithic integrated optic microchip for the measurement of the peak wavelength shift of FBG sensors exposed to passive and active events.

The integrated optic microchip technology enables the integration of all of the functionalities, both passive and active, of conventional bench-top FBG sensor interrogators systems, packaged in a miniaturized, low power operation, single fiber package suitable for the long-term structural health monitoring in applications where size, weight, and power are critical for operation.

\section{Acknowledgement}

Redondo Optics acknowledges the support of this work by the U.S. Navy under an SBIR Phase II Contract No. N68335-06-C-0049 under the program management supervision of Craig Lopatin.

Open Access This article is distributed under the terms of the Creative Commons Attribution License which permits any use, distribution, and reproduction in any medium, provided the original author(s) and source are credited.

\section{References}

[1] M. N. Trutzel, K. Wauer, D. Betz, L. Staudigel, O. Krumpholz, H. C. Muehlmann, T. Muellert, and W. Gleine, "Smart sensing of aviation structures with fiber-optic Bragg grating sensors," in Proc. SPIE, vol. 3986, pp.134-143, 2000.

[2] Z. Zhou and J. Ou, "Development of FBG sensors for structural health monitoring in civil infrastructures," presented at Proceeding of North American Euro-Pacific Workshop "Sensing Issues in Civil Structural Health Monitoring”, Waikiki Beach, Oahu, Hawaii, USA, Nov. 11-13, 2004.

[3] T. Bowker and M. Shaw, "Beyond telecom: FBGs find use in oil and gas industries," Laser Focus World, May, 2003.

[4] P. Moyo, J. M. W. Brownjohn, R. Suresh, and S. C. Tjin, "Development of fiber Bragg grating sensors for monitoring civil infrastructure," Engineering Structures, vol. 27, no. 12, pp. 1828-1834, 2005.

[5] M. Seaver, S. T. Trickey, and J. M. Nichols, "Strain measurements from FBGs embedded in rotating composite propeller blades," in Optical Fiber Sensors, OSA Technical Digest (CD) (Optical Society of America, 2006), paper ThD2.

[6] J. S. Leng, R. A. Barnes, A. Hameed, D. Winter, J. Tetlow, G. C. Mays, and G. F. Fernando, "Structural NDE of concrete structures using protected EFPI and FBG sensors," Sensors and Actuators A, vol. 126, no. 2, pp. 340-347, 2006.

[7] E. M. Dianov, "Fiber optics: from communications to 'nervous' systems," Herald of the Russian Academy of Sciences, vol. 77, no. 4, pp. 368-372, 2007.

[8] J. Hao, M. Jayachandran, N. Ni, J. Phua, H. M. Liew, P. W. A. Aung, J. Biswas, S. F. Foo, J. A. Low, and P. Yap, "An intelligent elderly healthcare monitoring system using fiber-based sensors," Journal of the Chinese Institute of Engineers, vol. 33, no. 5, pp. 653-660, 2010.

[9] DoD 2005 SBIR solicitation, "Miniaturization of a Fiber Bragg Grating Sensor Interrogator," Navy Topic No. N05-115.

[10] E. A. Mendoza, "Miniaturization of an optical fiber grating sensor interrogator," Navy SBIR Phase II Contract No. N68335-06-C-0049, 2007.

[11] J. Buswell, "Lessons learned from health monitoring of rocket motors," presented at $41 \mathrm{st}$ AIAA/ASME/SAE/ASEE Joint Propulsion Conference and Exhibit, AIAA 2005-4558, July 2005.

[12] E. A. Mendoza, "Fiber Bragg gratingsensor interrogator method," United States Patent, Patent 7,512,291, 2009 